\title{
CATACLYSMIC VARIABLES - CONCLUDING REMARKS
}

\author{
Z.DENĔK KOPAL \\ Department of Astronomy, University of Manchester, England
}

(Received 29 September, 1986)

Kitamura sensei, ladies and gentlemen: the remarks which follow will be the last communication by which our colloquium on cataclysmic variables is to be brought to its conclusion; and I appreciate the invitation of its organizing committee to use the remaining time we have together in the congenial atmosphere created for us at Bamberg, in an attempt at a summary of what we heard here in the past few days from several hundred colleagues who gathered here from many parts of the world - to survey the present state of our subject, and consider together where do we go from here. The time for this purpose makes it impossible to touch upon all exciting developments in the field of cataclysmic variables reported at Bamberg in the past few days; but certain general features have emerged from the programme of our colloquium which it may be appropriate to recall.

First, it is impossible not to note, nor stress enough, the novelty of the subject to whose discussion our programme has been dedicated. The subject is, in fact, scarcely more than one generation old; in the first half of this century our predecessors still knew next to nothing about cataclysmic variables of what we know today. The appearance of 'new stars' in the sky was, to be sure, noted from time to time (though a fundamental difference between novae and supernovae in different types of stellar populations was recognized by the middle of the century); but it was only afterwards that observations relating such phenomena with the binary nature of such objects began to emerge from newly accumulating observational data. When Nova Herculis flared up in December 1934 (a phenomenon which the present speaker already observed during his student years at the university), little did we dream that only a little more than twenty years later this particular post-Nova would be shown to be the component of one particular subdwarf binary (cf. Walker, 1956) - a discovery announced at the 9th General Assembly of the International Astronomical Union in Dublin where I was privileged to congratulate Dr Walker on his work from the chair of Commission 42 and - thus establish the first solid links between close binary systems and the Novae (represented up to that time in the IAU by Commission 27).

Nova Herculis 1934 (known since 1956 as eclipsing variable DQ Her) turned out to be the first cataclysmic variable (CV) which proved to be a close binary system; and its discovery spurred rapidly those of several other binaries of this type - such as GK Per

Paper presented at the IAU Colloquium No. 93 on 'Cataclysmic Variables. Recent Multi-Frequency Observations and Theoretical Developments', held at Dr. Remeis-Sternwarte Bamberg, F.R.G., 16-19 June, 1986. 
(N Per 1901), V 603 Aql (N Aql 1918), WZ Sge (N Sge 1913 and 1946) or T Aur (N Aur 1891); the last two of which turned out to be also eclipsing variables. Other CV's rapidly followed suit - including both prototypes of such semi-regular variables as SS Cyg or U Gem - both binary systems with periods of $6^{\text {h }} 38^{\mathrm{m}}$ for SS Cyg (cf. Joy, 1956) and $4^{\mathrm{h}} 10^{\mathrm{m}} 5$ for U Gem (Krzeminski, 1965); the second one of which also exhibits eclipses.

The reasons which the discoveries of binary CV's were so slow to come by (none was known in the first half of the 20th century) were primarily instrumental: virtually all of them are sub-dwarfs (and, therefore, apparently faint), requiring not only large-aperture optics for their discovery, but also fast-response photometers for discovery of rapid light variations - even today, with all modern technology at our disposal, it would still be very difficult to discover an eclipsing variable with a period of less than $1 \mathrm{~s}$ (let alone to establish the shape of its light curve!). Fortunately, however, other ways to discover various observational manifestations of different types of the CV's opened up in the past 10-15 yr with the advent of instruments lifted by spacecraft to orbital altitudes, at which much wider domains of the spectrum from the radio-domain to X-ray domain became accessible to direct observation.

A statistical analysis of the material submitted and discussed at Bamberg in the past four days discloses that approximately $86 \%$ of it predominantly dealt with the observational aspects of the CV's; and only $14 \%$ was concerned with their interpretation or theory; while at the last year's ESA Workshop on CV's, held likewise at Bamberg between 17-19 April, 1985 (with its proceedings published as ESA SP-236) almost $100 \%$ of the material under discussion concerned the observations: without reports on the contributions provided by the IUE, EXOSAT, EINSTEIN, and other satellites, almost the entire programme of that conference could have been presented in less than one day.

For the results of all these observations only admiration can be expressed; and the current progress augurs well for the future. However, when we pause to inquire about the meaning of all the new evidence which so suddenly fell into our laps, we find ourselves on much less secure ground. Let we mention, in this connection, a few facts which many of us must have found rather disquieting. Throughout the meetings, much of the observed data (both new and old) were presented to us on the screen in the form which statisticians would fairly describe as scatter-diagrams. Why should this be so? Are we, perchance, looking for relations between quantities which are physically unrelated, or only weakly related with each other? Are we, in brief, asking Nature the right questions, or merely 'beating about the bush'? We do not know yet the answer.

Another point - concerned now with the theory of the phenomena observed with such zest and skill. The most 'popular' geometrical configurations encountered on the screen in diagrammatic form these days are the 'Roche Lobes' - a trademark in our field, which could almost be worn as masonic pins to distinguish our identity. But - jokes apart these surely can serve only for decorative purposes; and as far as the CV's are concerned, are largely devoid of physical meaning. For, as is well known, the Roche lobes represent only equilibrium geometry of static double-star configurations, of negligible kinetic energy. But can the kinetic energy of the CV's be regarded as negligible? 
What is, in fact, the source of energy which may cause mass to be lost by their unstable components into space, or be (temporarily) retained by its mate in configurations commonly described as 'discs'?

Judging from the sense of our meetings, we are perhaps not too far from the truth with a conjecture that the principal mechanism of mass loss is the stellar wind: but what makes it occasionally in the CV's to blow with the strength of a hurricane? Is it powered by sub-surface convection, or a more deep-seated cause? And what is reponsible for the transient high-temperature phenomena observed occasionally in the CV's? Is the source of the requisite energy purely gravitational (through the medium of shock-waves), or chemical (nuclear)?

Turning to the 'rings' which (allegedly) surround the compact components of such systems, to say that these are unstable is only repeating the obvious. But how unstable are they - how long can the mass expelled from the companion linger in the equatorial plane of its compact mate before it becomes totally dispersed by internal pressure?

I am afraid that most part of this talk has so far been spent on raising questions to which we do not as yet know the answers. But to ask the right questions may, perhaps, be the beginning of the solution; for no problem can obviously be solved before it has been properly formulated; and this is the stage in which we find ourselves at the present time. Perhaps it is the observers who are partly to be blamed for this state of affairs; for - according to a theorem discovered some time ago our colleague (here present) Vanýsek, 'every new observational fact is inimical to some theory'. Perhaps our theories have lately received so many knockouts from unforeseen new facts to be reduced temporarily to a groggy state; but - let us hope - this state will not last for long.

For one of our staunchest allies in the near future - the theoreticians' answer to the observers' spacecraft - are the new-generation electronic computers of ever-increasing memory and speed, which may enable us to win the war against ignorance by sheer attrition - namely, of model-construction - some of which may eventually match the observed facts in their essential aspects. But these techniques too require caution. For no matter how many 'Cray-days' of computation we may be fortunate to get for our work, their prodigious speed and vast information storage are only ancillary for the quality and information content of the results. The latter will always be controlled by the intelligence which went into the writing of the programme to be carried out. An excellent technical performance of the computer is indeed necessary, but not yet sufficient, to ensure - not only the mathematical correctness, but also physical significance - of the results.

Yet - let us hope - our Cray computers of the future may outdo in performance the famous monkeys of James Jeans which set out to write Shakespeare's sonnets by random banging at their typewriters; and provide physically sound models of cataclysmic variables in the sky by sheer exercise of perseverance; and that - as a result when we meet again at the next colloquium in Bamberg devoted to our CV's, some of the scatter will have disappeared from the diagrams we have seen in the past four days; and the scattering of views we have heared expressed on their nature may be significantly lessened. 


\section{References}

Joy, A. H.: 1956, Astrophys. J. 124, 317.

Krzeminski, W.: 1965, Astrophys. J. 142, 1051.

Walker, M.: 1956, Astrophys. J. 123, 68. 